\title{
Real Instant Road Condition Monitoring With Data Logging Algorithm
}

\author{
Dr. D. Vishnuvardhan ${ }^{1}$, D. Sunitha Kumari ${ }^{2}$
}

\begin{abstract}
Most common problems that wear facing in our daily life is surface defects such as potholes, suck-in manholes, and defective street cuts are still commonly found on roads. Due to this current methods of road surface monitoring depends on reports of drivers. Conversely, these methods usually incur a long delay consequently it is difficult to obtain up-to-date information on road conditions. To conquer the drawbacks the current methods, we intend an innovative and automated method that uses mobile data collection kit mounted on vehicles. Here, we use a supervised machine learning technique to classify the collected data fragments into three different categories at a back-end server. The mobile data collection kit includes accelerometer, global positioning system (GPS) sensor and microcomputer. To increase the efficiency of data logging a local algorithm is embedded. Large amount of collected data are transmitted to a back-end server.
\end{abstract}

Keywords: Road condition monitoring, Data logging algorithm, Accelerometer, GPS

\section{Introduction}

Road condition monitoring in real-time system is based on systematize process of data logging. Earlier, the road surfaces are tranquil not well maintained, having defects such as potholes, suck-in manholes, and defective street cuts. So, due to these poorly maintained roads can damage vehicles and furthermore they can cause life threatening accidents. In past time taken manual method was used, nowa-days we are using 'ARM7 based technology'. By this technology we progress a smart road surface monitoring method. The advantage of this method is that a large range of roads can be monitored simultaneously by simply mounting the kit onto normal vehicles. The collected data are transmitted to a back-end server, where the road surface algorithms are usedto identify road conditions based on the data.

\section{Existing Method}

The architecture of existing road surface monitoring is based on local database. It consists of two parts: a vehicle client (i.e., a mobile data collection kit that can be mounted onto vehicles) and a back-end server. In each vehicle client, an accelerometer, GPS sensor, microcomputer, and local storage are included. Because a vehicle client could not always have Internet connection, the composed data are first cached on the local disk. So the data were collected with a high frequency and the majority of the data are collected on smooth roads.

\section{Proposed Method}

The architecture of our proposed road surface monitoring system is illustrated in figure shown below

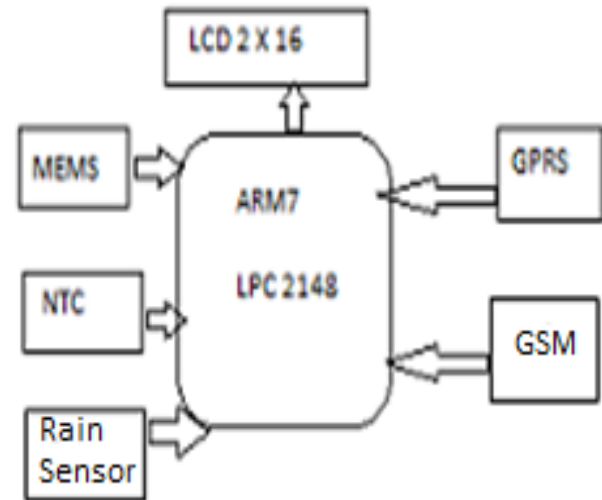

Figure: Architecture of proposed road surface monitoring system.

It consists of two parts: a vehicle client (i.e., a mobile data collection kit that can be mounted onto vehicles) and a backend server. In each vehicle client, an accelerometer, GPS sensor, GPRS, NTC sensor, Rain sensor, MEMS are included. We use a data logging algorithm that runs locally on each vehicle client to filter out unnecessary data that are collected on smooth roads.

\section{Data logging Algorithm}

Logging all of the measured data into the local storage cannot be very efficient because only some of them contain useful information to detect smart road exterior defects. As an illustration, data recorded on smart smooth roads do not have significant excitation in measured signals and just take extra storage spaces. We design a data logging algorithm that runs in each vehicle client in order to increase the competence of the data logging. The data logging algorithm records the data only such as taking turns, changing lanes and going up and down hills.

In current method we use a supervised machine learning technique to classify into different categories from collected data fragments in at back-end server. These categories are consequently dissimilar. In this the first category is impulse class, where we observe sudden vibrations. Second category is rough class, disparate impulse responses; a long period of 


\section{International Journal of Science and Research (IJSR) \\ ISSN (Online): 2319-7064}

Index Copernicus Value (2013): 6.14 | Impact Factor (2015): 6.391

vibrations is shown due to the roughness of road surfaces. Continuous defective street cuts and bumpy roads account for this class. Third category is smooth class. In this class, no signification vibration is accessible in acceleration response. We use a multilayer feed-forward neural network to categorize the composed data fragments into three classes.

\section{System Structure}

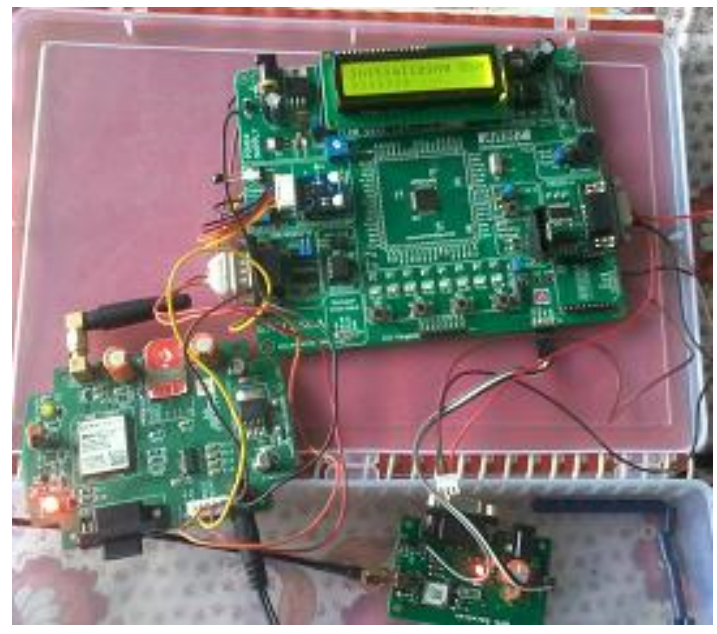

Anew road surface monitoring method, which uses a mobile data collection kit. This kit used for collect data regarding road surface conditions.

\section{System Hardware}

Hardware components includes ARM7, LPC2148, LM11173.3, 2X16 LCD Display, Bridge Rectifier, M95, GSMModem, GPSMODEM, MEMSSENSOR, ADXL335,NTC,RAIN, SENSOR,MAX 232.

\begin{abstract}
ARM7
ARM is an Advanced RISC Machine. ARM is one of the most approved and therefore widespread processor cores in the world. The ARM7TDMI processor has two operating states:

ARM - 32-bit, word allied ARM instructions are performed in this state. Thumb -16-bit, semi word-aligned Thumb instructions are performed in this state. The functioning state of the ARM7TDMI core can be substituted among ARM state and Thumb state by means of the BX (branch and exchange) orders. The ARM7TDMI-S is a universal purpose 32-bit microprocessor, which offers high presentation and very low power consumption.
\end{abstract}

\section{LPC2148}

LPC2141/42/48 microcontrollers are based on a 16-bit/32bit ARM7TDMI-S CPU with real instant emulation and embedded trace sustenance, that syndicate microcontroller with embedded high speed flash memory fluctuating from $32 \mathrm{~KB}$ to $512 \mathrm{~KB}$. Due to their low power and tiny size consumption, LPC2141/48 are model for applications where miniaturization is a key requirement, such as access control and point-of-sale.

\section{LM1117 3.3}

The LM1117 is a series of low dropout voltage regulators with a failure of $1.2 \mathrm{~V}$ at $800 \mathrm{~mA}$ of load current. It takes the similar pin out as National Semiconductor's industry standard LM317.The LM1117 is accessible in an adaptable version, which can usual the output voltage from $1.25 \mathrm{~V}$ to $13.8 \mathrm{~V}$ by only two exterior resistors. In adding, it is also obtainable in five static voltages, $1.8 \mathrm{~V}, 2.5 \mathrm{~V}, 2.85 \mathrm{~V}, 3.3 \mathrm{~V}$, and 5V. The LM1117 suggestions existing limiting and thermal closure.

\section{LCD DISPLAY}

The term liquid crystal is used to describe a substantial in a state between liquid and solid then which displays the properties of both. Molecules in liquid quartzes slope to position themselves until they all point in the similar specific direction. This arrangement of molecules enables the intermediate to flow as a liquid. Dependent on the temperature and specific nature of a substance, liquid crystals can occur in one of several different phases. Molten crystals in a pneumatic phase, in which there is no spatial ordering of the particles, for example, are cast-off in LCD technology. One important of liquid crystals is the fact that an electrical current affects them.

\section{GSM}

The GSM specifications define the purposes and boundary requirements in aspect but not the address the hardware. The reason for this is to bind the designers as slight as possible but it static to make it probable for the operators to buy equipment form different suppliers. The GSM network is divided into three key systems: The Switching Scheme (SS); The Base Station Scheme (BSS); and the operation and Support Scheme (OSS).

\begin{abstract}
ADXL 335
The ADXL335 is a minor, reedy, low power, complete 3 axis accelerometer through signal trained voltage outputs. The product processes acceleration with a minutest full-scale range of $\pm 3 \mathrm{~g}$. It can amount the static acceleration of gravity general tilt-sensing applications, as dynamic acceleration resultant as of motion, shock, or vibration. The sensor stands a polysilicon surface-micro machined structure built on highest of a silicon wafer. Polysilicon springs hang the structure ended the surface of the wafer and offer a resistance next to acceleration forces. Deflection of the structure is leisurely by a differential capacitor so as to consist of sovereign fixed plates and plates devoted towards the moving mass. The fixed plates are resolute by $180^{\circ}$ outof-phase square waves. Acceleration bounces the moving mass and interrupts the differential capacitor subsequent in a sensor output whose amplitude is comparative toward acceleration. Phase responsive demodulation techniques are next used to conclude the magnitude and direction of the acceleration.
\end{abstract}

\section{NTC}

NTC temperature sensors are entire from a mixture of metal oxides which remain exposed to a sintering process that stretches them an undesirable electrical resistance against temperature. NTC temperature sensors are broadly used in motor vehicles. This variety of best glass encapsulated. NTC temperature sensors are price modest for general use. Not 


\section{International Journal of Science and Research (IJSR) \\ ISSN (Online): 2319-7064 \\ Index Copernicus Value (2013): 6.14 | Impact Factor (2015): 6.391}

only can the leaded sensor be use at up to $300{ }^{\circ} \mathrm{C}$, except their glass encapsulation brands them ideal for use in harsh atmospheres besides harsh environments. This brands them and keen additional to other more exclusive sensing methods.

\section{MAX232}

The MAX232 stands a dual driver/receiver that contains a capacitive voltage producer to source TIA/EIA-232-F voltage levels since a single 5-V supply. Each receiver alters TIA/EIA-232-F inputs to 5-V TTL/CMOS levels. These receivers have a characteristic threshold of $1.3 \mathrm{~V}$, a illustrative hysteresis of $0.5 \mathrm{~V}$, in addition can receive \pm 30 $\mathrm{V}$ contributions. Every driver changes TTL/CMOS approximately levels addicted to TIA/EIA-232-F levels. The recipient, driver, and electrical energy generator purposes are accessible as cells in the Texas Instruments Lin ASI $\square$ library. Meets or Exceeds TIA/EIA-232-F in addition to ITU Recommendation V.28. Operates As of a Single 5-V Power provide through.0-F Charge-Pump Capacitors. It operate Up To $120 \mathrm{kbit} / \mathrm{s}$.

\section{Result and Conclusion}

This developed program is successfully burned on the ARM7 kit using USB programmer. Collect the road surface monitoring kit and set up the connections appropriately. Initializing GSM and GPRS is done through LPC2148.
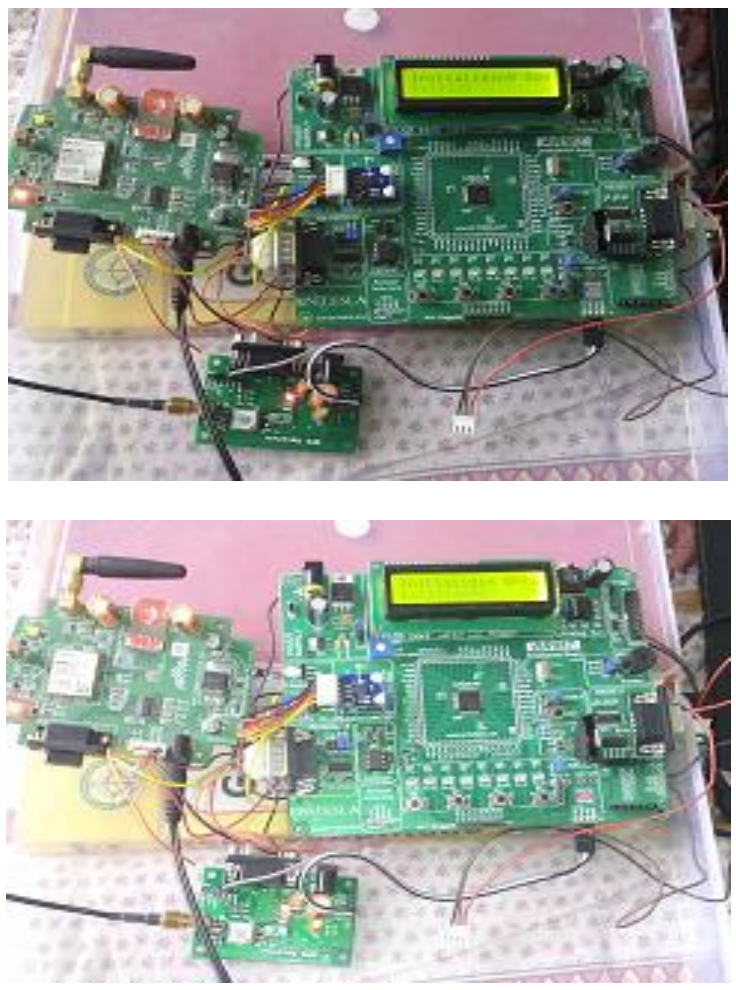

To find out smart road condition we use different multiple sensor- equipped vehicles to automatically identify road surface conditions, and veteran it on real roads. To automate the process of collecting the information, we developed a new real time road condition monitoring method, which uses a mobile data collection kit mounted on vehicles to collect data. To find out smart road condition we use different multiple sensor- equipped vehicles to automatically identify road regarding condition and practiced on real roads

The advantage of this method is so as to a huge range of roads can be monitored simultaneously by simply mounting the kit onto normal vehicles.

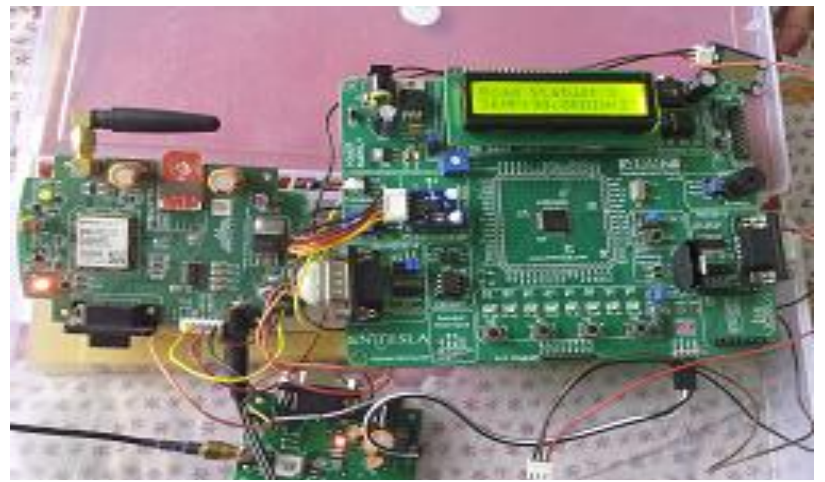

After initialization it detects the road condition and also it detects the location with the help of GPS.

It display the road condition and its location where we moving towards forward with the help of Liquid Crystal Display (LCD).

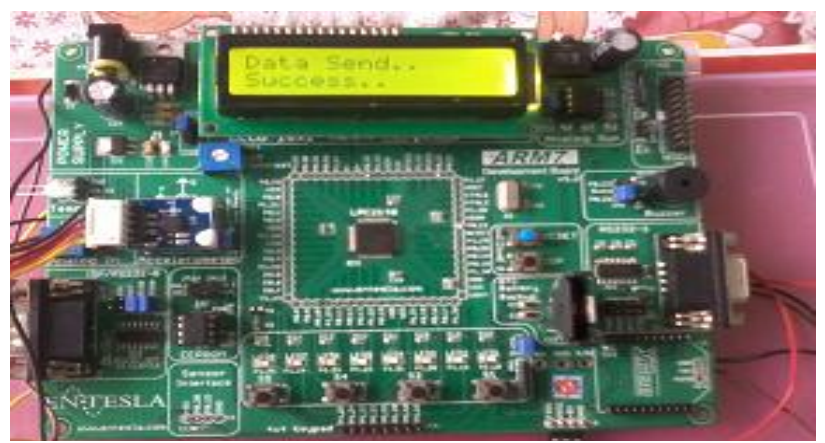

If it display as data send success then only the signal rate denotes as good. In each vehicle client, an accelerometer, GPS sensor, microcomputer, and limited storage are included. Logging all of the measured data into the local storage cannot be extremely efficient because only some of them contain useful in order to detect road surface defects.

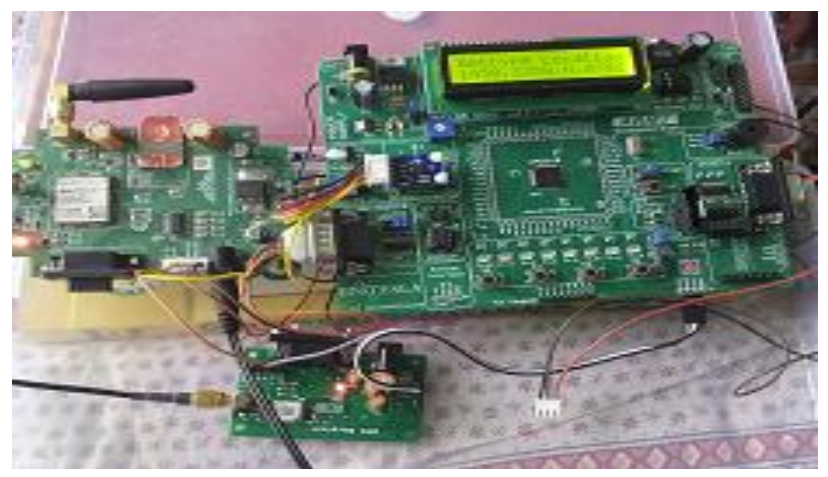

Volume 5 Issue 6, June 2016 www.ijsr.net 


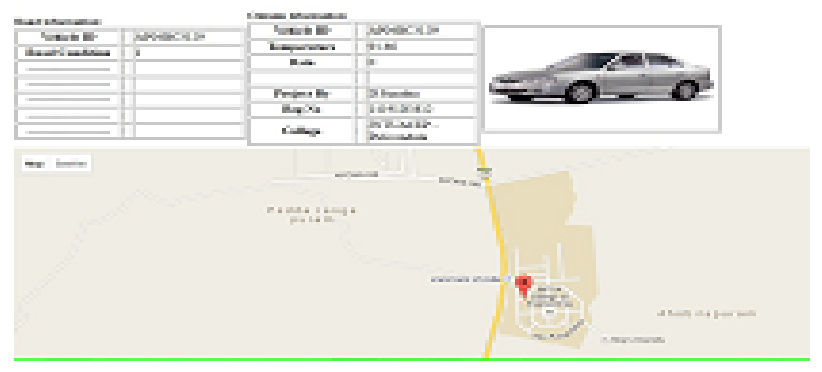

Here we introduce Road Mapping System to show the distance as well as the place where we have to reach safely. This Road Mapping System is detected with the help of GPRS .Based on signal level we detect Road Condition.

To detect the Road condition we can perform on Real Time Smart Road Light Mapping System.

\section{References}

[1] Smart Growing America and Taxpayers aimed at Common Sense, "Repair Priorities 2014: Transportation outlay strategies to save taxpayer dollars next progress roads," Nov. 5, 2014. [Online]. accessible: http://www.inevolutionamerica.org/documents/priorities2014.pdfs

[2] American origin of collection Engineers, "2013 declaration qualification for America's infrastructure,"Nov.5, 2014, 2013. [Online].obtainable: http://www.infrastructureassertioncredential.org

[3] L.Girod,B.Hull,J.Erikson,R.Newton,S.Madden , and H. Balakrishnan, 'The pothole patrol: by a mobile sensor network for Smart Road Condition monitoring,' in Annals of the 6th world wide discussion on Mobile systems, applications, and services. ACM, 2008, pp. 2939.

[4] J.-G. Lee J. Han, and K.-Y. Whang, "Route congregation: adivider and collection framework," in Records of the 2007 ACM SIGMOD world wide meeting on Management of data. ACM, 2007, pp. 593-604.

\section{Author Profile}

Dr. D. VishnuVardhan, Assistant Professor in the Department of Electronics and Communication Engineering, JNTUA College of Engineering, Pulivendula. Y. S. R (Dist). A.P (State).Pincode:516390.

D. Sunitha Kumari, M.Tech in Digital Electronics and Communications Systems, JNTUA College Of Engineering, Pulivendula. 